\title{
Simplified for Resilience: \\ A parametric investigation into a bespoke joint system for bamboo
}

\author{
Carrie Wendt Matson \\ Victoria University of Wellington, New Zealand \\ redmanatee@gmail.com \\ Kevin Sweet \\ Victoria University of Wellington, New Zealand \\ kevin.sweet@vuw.ac.nz
}

\begin{abstract}
Research reveals that most of the structural failures in a natural disaster are related to improper construction assembly methodologies related to human errors. This paper aims to reduce human errors in the building process by taking advantage of computational tools, and using a renewable building material.

The research investigates the creation of a novel structural system for bamboo that is able to be repaired, replaced, altered, and easily assembled to restore any damaged building structure. Bamboo is an organic product with diameters that are irregular and unpredictable. The inconsistency in this natural product requires an adaptable construction methodology that responds to its organic nature. A customised joint system is created using parametric software that quickly adapts to the irregularity of the bamboo and are then fabricated using additive printing techniques. The parametric software gives unlimited control of the joint system based on the programmed relationships between the differentiations of each unique bamboo connection. Fabricating each unique joint gives a secure connection at each intersection facilitating an adaptable architecture, whilst reducing construction waste.

This paper introduces the groundwork for the implementation of "on-site" manufacturing of a framework joint system. The manufacturing utilises the power and performance of a parametric platform with the technology of bespoke three-dimensionally printed joints - a flexible system that can respond to organic materials and natural external conditions
\end{abstract}

Keywords: Parametric design; Three-dimensional printing; Bamboo construction.

\section{Introduction}

Extreme natural disasters cause catastrophic damage to the world's built environment putting both people and property at risk. The ever-increasing population means more houses and buildings are being destroyed than ever before (Creel, 2003). Every natural disaster involving the built environment has devastating consequences not only to property but also to the psychology and well-being of the inhabitants. After each disaster, there is grief for loss of life, loss of personal belongings, and a loss of place. Rebuilding damaged buildings can quickly revitalise a city, the sprit of its people, and aid in alleviating the community's distress. Rebuilding gives hope to the people, provides them with a future, and helps with working through the grieving process (Kerr, 2012)

Ideally, rebuilding occurs as quickly as possible. However, Christchurch, New Zealand is a perfect example of how difficult it is to rebuild and how long it can take. Over six years following the earthquake of 2011, Christchurch is still struggling to rebuild itself and the houses of many residents (Insurance Council of New Zealand, 2016). Experiencing a natural disaster coupled with rebuilding personal lives creates stress and financial pressure on affected communities: many take years, even decades, before rebuilding is complete and the cost can be financially crippling (Indrawati \& Steer, 2005).

Building is a complex ordeal and many technical errors can be made (Suaris \& Khan, 1995). Building methodologies need to change in order to reduce the potential for error and create a simplified efficient solution. Structural configurations, materials, and the assembly process of a building are all part of the problem. These elements need to be reconsidered in a new way, if a productive solution is going to be found.

The paper concentrates on creating a customised structural system using parametric design techniques intended to develop a new building methodology that will allow construction to be built or altered quickly and efficiently with very little product waste. The new building model explores a 
simpler, stronger, more robust joint system using bamboo. The use of parametric software facilitates a range of variables to customise the design of the structural system. Parametric design also expedites the manufacturing process by closing the gap between the virtual and the physical, subsequently facilitating a less complicated construction methodology (Tibbits, 2015). The design uses tailor-made joints for bamboo canes that are parametrically created and physically fabricated using additive manufacturing. The relationship of parametrics with three-dimensional printing creates a seamless progression from the virtual world to the physical world. This innovative design-print-assemble construction method creates a direct relationship between the designer and the architecture, resulting in a simplified building process. The materiality of the joint system can vary, as can the structural requirements and aesthetics of a specific project.

\section{Significance of Parametric Design in the Construction Process}

The significance in choosing a parametric design process was to create a flexible construction method facilitating an adaptable architecture. Building flexibility into the design enables materials of various shape and sizes to be used. Because bamboo is variable and imperfect, the joint system needs to adapt to individual pieces of bamboo to make the structural system function. Parametric design gives the ability to change and adapt with each cane. Relationships established in the design allow for modifications of the dimension of the joint to fit the bamboo and for other programmed purposes such as aesthetics or structural integrity. The connecting joints are designed around the organic material rather than the material being altered for the joint. The parametric approach opens up flexibility that does not exist in a conventional construction processes and allows for organic rather than mass-produced materials.

In addition to the ability to alter the digital model's dimensions easily, the parametric approach gives a fluent conversion between the virtual and the physical. The parametrically designed construction joint responds and adapts to the material as required. The digital model can be altered and printed to produce a working construction joint in minutes. The efficient workflow from digital design to fabrication enables the joint to be tested on the bamboo for a secure fit and assembled immediately after printing. This direct construction process simplifies the steps of building lowering the potential for human error. Fabricating customised construction products directly from their digital model results in little to no waste. The organic materials can keep their natural form, rather than being milled, or altered for the building process (Kendell, n.d.). The ability to change the design creates a responsive and adaptive architecture that can be rebuilt, repaired and changes its overall shape and configuration (Kolarevic, 2015). "The idea that [the] built environment is in constant transformation and change must be recognized and understood" (Kendell, 2004, pg.92)

Digital design is a generative tool not just a visual one, and the outputs are able to manifest themselves in more complicated and specialised constructions (Kendell,n.d.). This parametric design generates sophisticated forms while maintaining simplicity of assembly. The ability for a designer to deliver a design from a virtual model and printed physical form is a simple and flexible methodology. Kronenburg (2015) wrote about the reasons flexible architecture is needed. He said that flexible architecture is fit for purpose with the ability to modify, if the programme changes. This enables the building to be used for longer by responding to the owner's needs (Kronenburg, 2015)

\section{Design Methodology}

The design methodology was broken in to three phases: Phase One was research and case studies; Phase Two was a simplified design with scaled down prototypes of the joints using standard thin bamboo canes; and Phase Three designed $1: 1$ scaled joints using $80 \mathrm{~mm}$ and $40 \mathrm{~mm}$ diameter bamboo

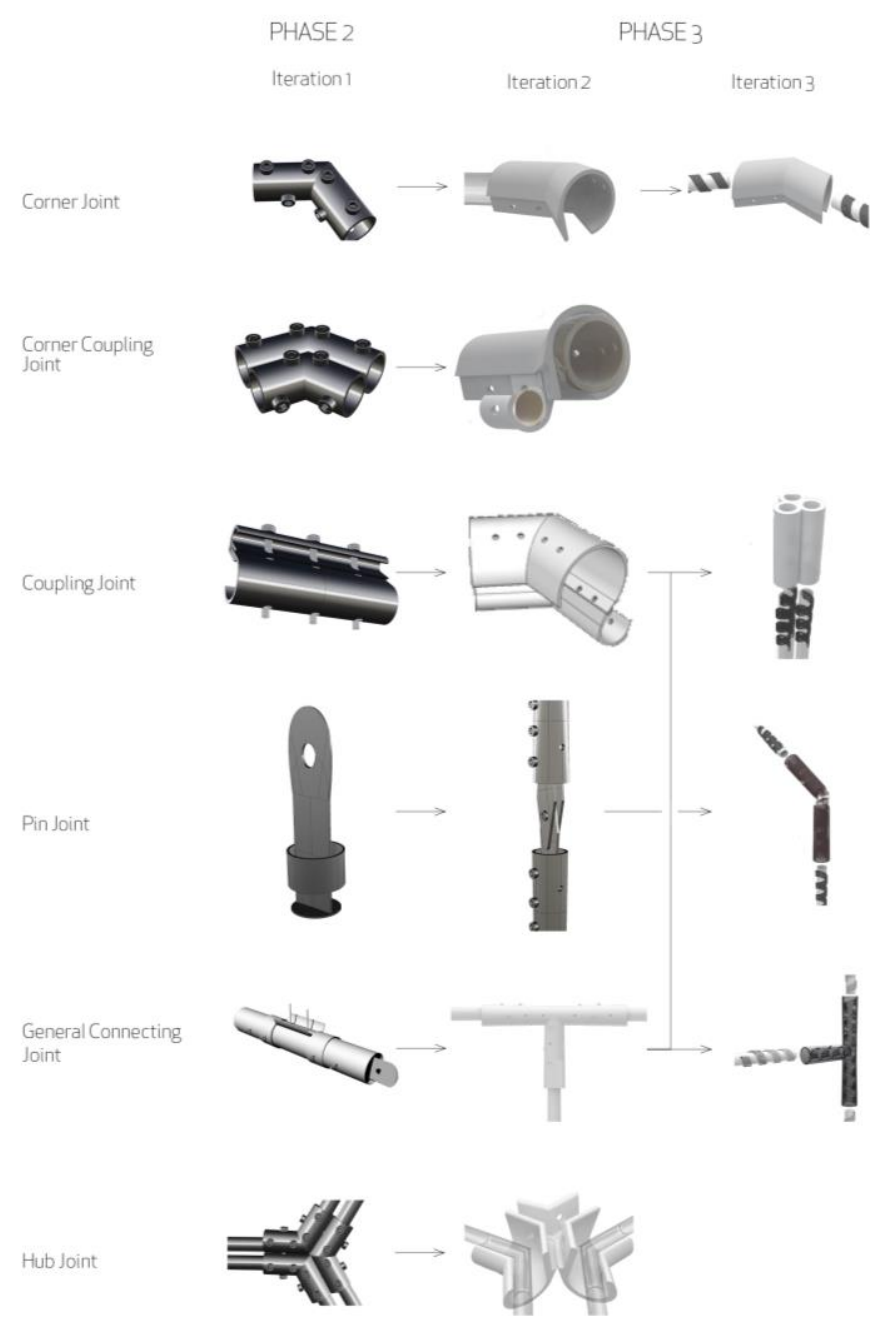

Figure 1: Digital model iterations from Rhino to Digital Project 


\section{Phase One: Case Studies}

During phase one, the case studies research was broken up into two categories; first the assessment of timber structures that failed in natural disasters and analysing why they failed. The second case study group was the research of bamboo structures and examination of the joints that make up the intersections for these structures.

The first case study group revealed the structural failures of timber dwellings in a natural disaster are caused predominately from the incorrect assembly of the intersections. Based on this fact, it was derived that the new design methodology needed to negate any deviation from the proper assembly process. The structure also needs to have the ability to be rebuilt quickly after a catastrophic event.

The second case study group examined precedents for various types of joint systems that have been used on existing bamboo structures. These joint systems illustrate endless variations of assembly processes that are possible. The amount of variations emphasises the need to design a consistent assembly process within a single structure. The joints will need to adapt and develop but the assembly should be a fixed process.

\section{Phase Two: Scaled Prototypes}

The design began with a set of criteria based on the research and case studies from phase one. The primary criteria stipulated that the joints had to have a clear and concise process of assembly that was simple to understand and implement. In addition, the joints could not weaken the integrity of the bamboo or compromise the strength of the overall structure in anyway.

The first design iterations were a series of hollow cylinders created with Rhino 3D modelling software. The bamboo simply slipped into the joint and was fixed to the bamboo with screws. The screws penetrated through holes designed in the joint and pre-drilled holes in the bamboo. This configuration worked well in the digital model but when the design was threedimensionally printed and assembled, the issues with the nonparametric software became evident. The lack of parameters required models to be rebuilt every time there was a design change and the model had to be built to tight tolerances in order to achieve success in physically printing it.

The assembly was arduous, drilling holes through the bamboo canes proved challenging. The round sections of the bamboo tended to move while being drilled, mainly due to the nods causing irregularity in the shape. It was difficult to secure the bamboo gently without damaging it. This caused inappropriate angles that did not align with the openings in the joint. This became a forced alignment creating tension in the bamboo, or a complete failure. The simple solution was to move the holes in the digital design to fit the holes in the physical bamboo and reprint the joint, but this was even more arduous as the model had to be rebuilt every time. There was no relationship to anchor the digital model's holes with the physical holes in the bamboo. It became a 'hit and miss' process based on a guessing game. A process mirrored in the attempt to adapt the joint to the bamboo's actual dimensions. Each piece of bamboo is unique with varying node diameters and cane taper. Attempting to respond to the bamboo's form using Rhino was inefficient and non-productive. The joint had to be completely remodelled with every little change, and with constant remodelling the geometry would become corrupt and causing the physical print to fail. The complete design process was not working; from the relationships with the bamboo to the relationship with $3 \mathrm{D}$ printing technology. There needed to be more flexibility in the design process and the printed joint needed to cater for a range of cane dimensions. The interchange between the design platform and the $3 \mathrm{~d}$ printing technology had to be resolved in the next phase.

\section{Phase Three: Full Scale Production}

The primary focus of phase three was to find a fluent discourse between the virtual and the physical. Parametric software was introduced to create a solution for the unreliable communication between the non-parametric software and the 3D printer. It also supported flawless geometry that is needed for physical fabrication. Gehry Technologies Digital Project was the platform used to parameterise the model and improve the 3D printing capability. Implementing parametric software was a successful process for the creation of the digital geometry and resultant physical fabrication of the joints. Using a parametric platform created a seamless transition for the digital to the physical. This was a major increase in productivity and success but in order to introduce the level of flexibility needed in the design process, the digital model needed to adopt the parametric ideology of relationships between geometries.

This was the next step in the design evolution. The programming of parametric relationships allowed for the alteration of geometry without changing the joint design or the overall aesthetic of the architecture. Establishing relationships facilitated the use of organic materials more efficiently. The nature of organic materials is imperfect and the ability to create tailored-made joints for each individual cane ensured a secure connection. With the establishment of a successful fabrication process and a functional parametric model, the work could focus on achieving the established criteria from phase one. The current design lacked ease of assembly and compromised the bamboo's strength with the pre-drilled penetrations. The design required a simpler and more concise method of assembly without the need for penetrations in the bamboo.

\section{Fabrication and assembly}

The power of the new parametric platform promoted a design that was responsive to the bamboo and adaptive to the configuration of the architecture. Using these strengths, the iterations that followed used a helix system to assemble the bamboo to the joint. An individual helix was fabricated and fixed to the bamboo cane and an identical helix was fabricated inside the corresponding joint. The two pieces connect together like a giant screw and nut. The helix system creates a clear and simple assembly process and does not tolerate deviation from the goals of the assembly or the intersection of multiple bamboo pieces. The joint design dictates the intersection's arrangement and the direction of the bamboo canes. All the flexibility is embedded in the design stage. With a parametric platform, the flexibility is instant and enables a transformable architecture. 
PHASE 2

Fabrication 1

Fabrication 1 tested the digital model's design

$30^{\circ}$ Corner Joint

$60^{\circ}$ Corner Joint

Corner Coupling Joint

Coupling Joint

Pin Joint

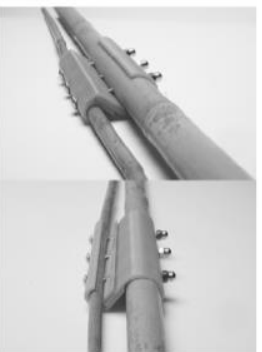

The coupling joint was designed to combine the corner joint and the coupling joint. This allows for more bamboo to be exposed less joints.
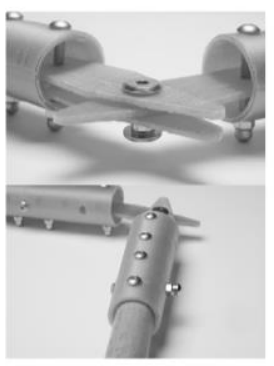

\}

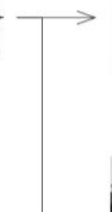

Fabrication 2 was to intended evolve the shapes to improve the functionality.

\section{PHASE 3}

Fabrication 2

Fabrication 3

Fabrication 3 was to intended evolve the design further and find the most functional assembly process,
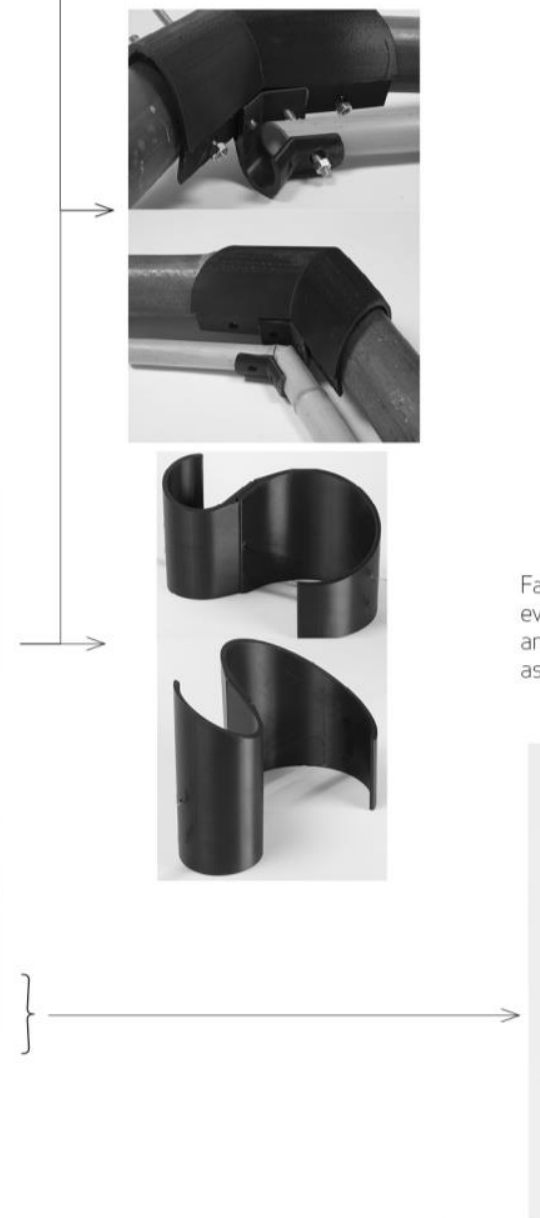

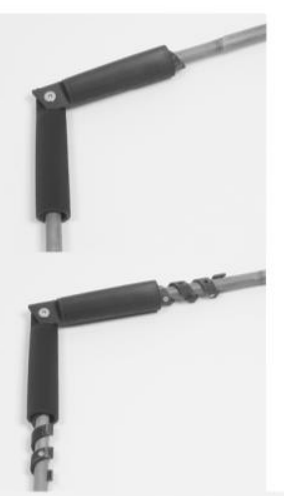

General Connecting Joint
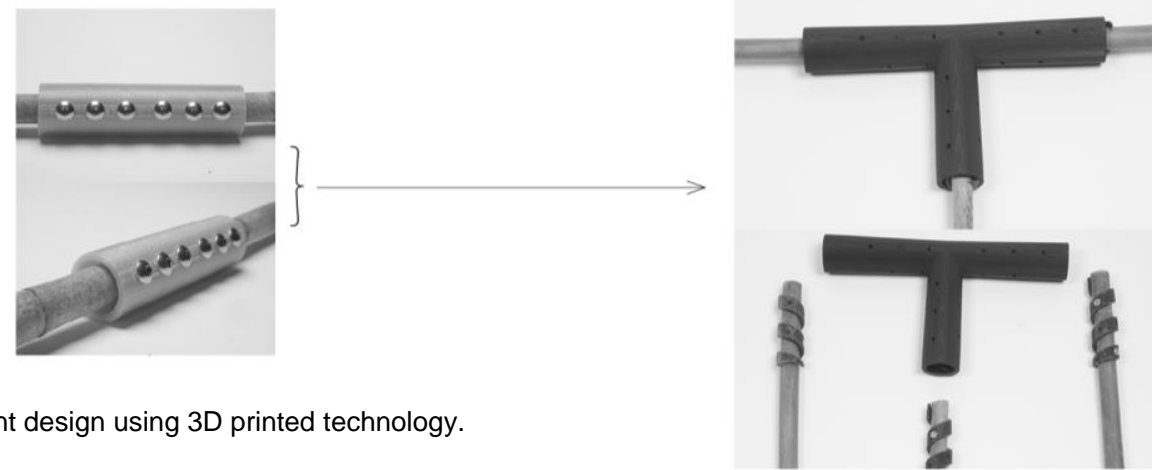

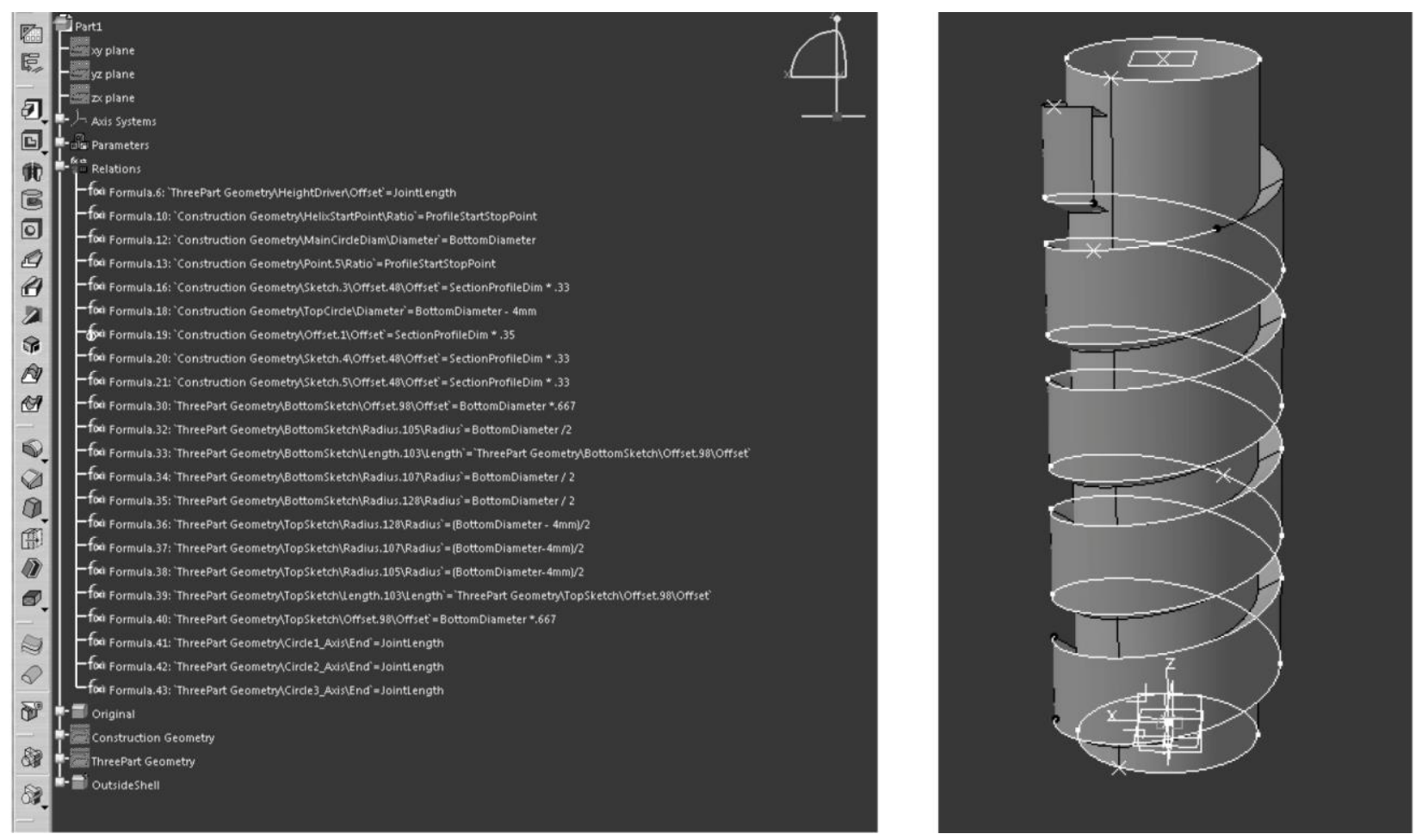

Figure 3: Digital Project relationship formulas for a helix joint

Relationships that are established in the parametric model enable flexibility in the design but in order to facilitate these relationships, sequence of the design needs to be carefully considered. Because there is no opportunity to alter the joint once it is printed, the digital model must be designed to completion. The bamboo must also be physically present for the joint to be tailored-fitted for each individual cane. The canes are measured and the dimensions inserted into the appropriate parameter of the model. Once the measurements are input, the joint design is customised for a specific bamboo cane. The joint is now ready for the production process. The model is able to be 3D printed on the building site and the joints are instantly ready for assembly. Joints that need alteration or repair, can be changed in the model and reprinted. Damaged bamboo canes can be replaced, without disrupting the overall structure. This was a low-tech assembly method facilitated by a high-tech design process.

This construction process has an additional benefit of a reduction in product waste for the overall construction process. All the construction components are printed and fitted for purpose onsite. In New Zealand, Construction and Demolition, waste accounts for up to $50 \%$ of all generated waste (Greater Wellington Regional Council, 2012). New initiatives are being actioned globally to reduce construction waste. This is a reaction to a long-standing problem. This new construction model will empower a proactive solution.

\section{Parametric affect}

The flexibility inherent in parametric design future-proofs the building, giving the opportunity for the architecture to evolve as the programme of the building alters or occupant's needs change. Parametric design enabled a seamless transition from a digital design to a physical component. The parametrics create a precise digital image that is easily manufactured and facilitates a simple methodology, ease of assembly and responsiveness to the organic material (Kolarevic, 2015). Parametrics is the single contributing factor in the flexibility and precision of the design. This level of flexibility and precision of the digital model was not achievable in other design process. The flexibility gives choices to the designer and supports last minute alterations or changes in material. The bamboo joint system is designed around the architecture allowing the building to respond and adapt to current environmental demands. "There are emerging opportunities to rethink the ways that we collaborate with materials and the way that we assemble components" (Tibbits, 2015 pg146).

\section{Conclusions}

This research introduces the idea of a changeable structure using a parametrically designed joint system fabricated by three-dimensional printing. The ethos of this design is adaptive, resilient, responsive, and robust architecture. This is achievable by utilising a changeable structural system with a rigid assembly process. The research proposes a construction process that starts in the design phase and is a fluid exchange of information transferring ideas into a digital relationship between the parametric model and the 3D printed technology

This design solution creates an unrestricted responsive bamboo structure with a unique connecting joint system. The structure has the potential to be changed or repaired by printing new components and replacing the bamboo canes. The spatial arrangements can be redefined and the structure can adapt to environmental conditions or transformed to respond to future demands. 


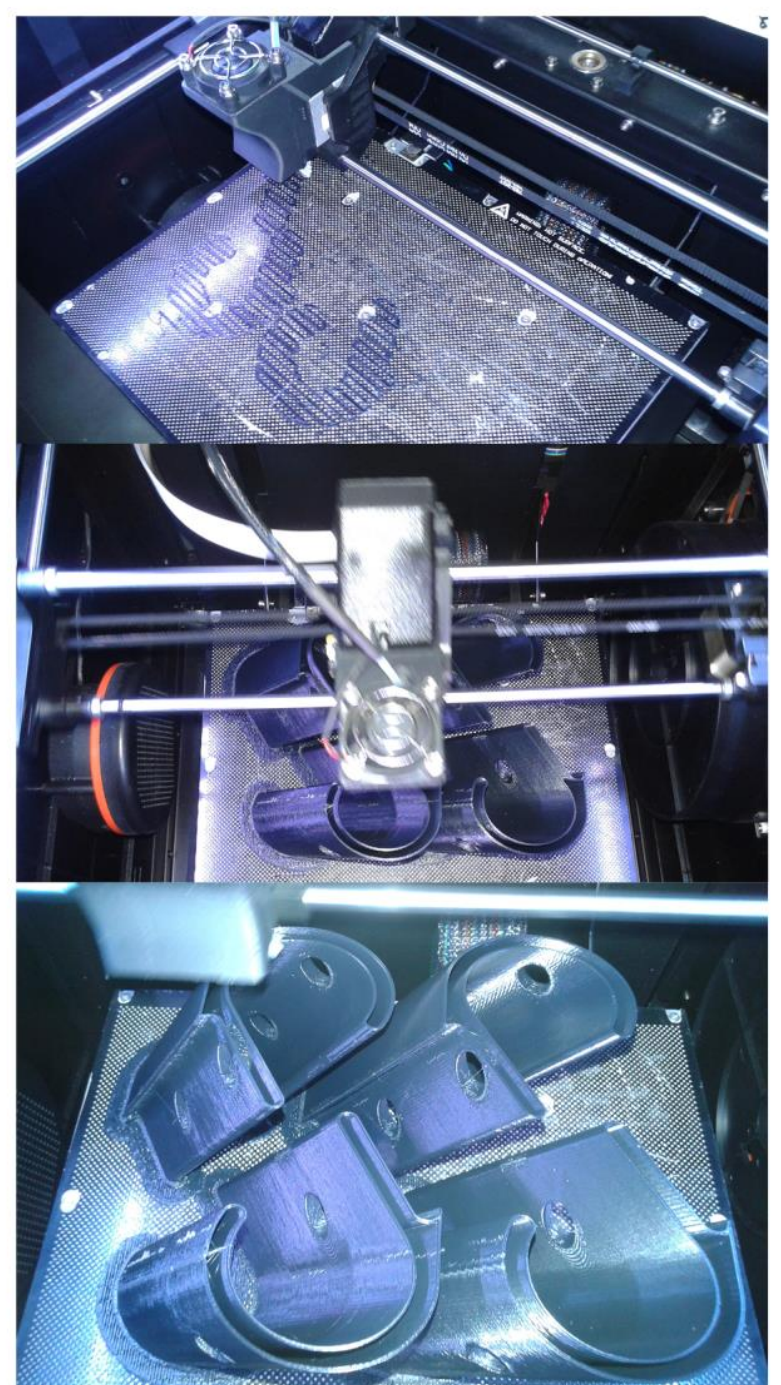

Figure 4: 3D printing 1:1 scale joints for fabrication run 2.

Kolarevic (2015) writes about the evolving facades of the 1990's, but over the last decade the focus has turned to dynamic structures that change their overall shape and provide different programmatic arrangements (Kolarevic, 2015). Forcing conventional building materials to conform to a new idea of architecture is ineffective. Integrating adaptability into a structure is the challenge architects face in the future (Kolarevic, 2015). Changeable architecture is the product of flexible design. The ability to accommodate change or have a dynamic configuration of spaces originates from the components that make up the structure. The components must have the same capacity for change expected of the overall architecture. The changeability designed into components uses parametric relationships to control the modifications. Creating a fabricated flexible hardware to configure a resilient and adaptable is an efficient use of materials, time, and space and is one way forward for architectural design and construction processes.

One challenge that has been foreseen is the sequence of the physical assembly process. Assembling the last cane of bamboo in a closed configuration remains unresolved but there are a variety of precedents to base a solution on. The issue is surmountable by experimenting with various assembly combinations. The established parametric design process is flexible enough to accommodate any alterations and resolution of the issue will come with a trial and error process.

It is also noted that further research into the longevity of bamboo as a structural material, must also be considered for future work. The cellular makeup of bamboo absorbs moisture and makes it vulnerable to insects. There are documented methods of preservation but finding a method that gives extensive life to the bamboo is crucial to support this robust design philosophy ('Bamboo as a building material', n.d.). It is not in the scope of this research, but ideally, an eco-friendly sustainable treatment would be readily available and create longevity without compromising the strength of the structure.

The presented research is preliminary; the physical assembly is well tested with fabricated prototypes of individual joints, however a complete structure has yet to be assembled. Regardless, the future of this design is very positive. This adaptive and simple infrastructure opens up numerous opportunities. The form and scale of the building can be determined based on the need and budget of the occupant and can change with trends. Flexible or changeable architecture allows a building to be used longer, eliminates obsolescence, and enables modifications as their purposes change in response to users' needs (Kronenburg, 2015). This building process has numerous applications; the quick and easy assembly process empowers the occupant to construct or repair their building themselves, the design can be used for disaster relief accommodation or residential dwellings, and can facilitate a timely rebuilding after a natural disaster. The parametrically designed connective joint system opens up opportunity into new construction methods for an adaptable robust architecture. 


\section{References}

Bamboo as a building material. (n.d.). Retrieved 15 October 2015, from http://bambus.rwthaachen.de/eng/reports/buildingmaterial/buildingmaterial.html

Creel, L. (2003). Ripple Effects: Population and Coastal Regions. Retrieved $13 \quad$ February 2016, from http://www.prb.org/Publications/Reports/2003/RippleEffectsPopul ationandCoastalRegions.aspx

Greater Wellington Regional Council. (2012, June 20). Construction waste | Greater Wellington Regional Council. Retrieved 13 June 2016, from http://www.gw.govt.nz/construction-and-demolitionwaste/.

Kendell, S. D. (n.d.). Open Building Concepts. Retrieved 20 February 2016, from http://open-building.org/ob/concepts.html

Kendell, S. D. (2004). An Open Building Strategy for achieving dwelling unit autonomy in multi-unit housing. House and Society, 31(1), 89-99.

Kerr, L. K. (2012, November 1). Grief Following Natural Disasters. Retrieved $26 \quad$ June 2016, from http://www.laurakkerr.com/2012/10/31/grief-following-naturaldisaster

Kolarevic, B. (2015). Building Dynamics: Exploring Architecture of Change. (V. Parlac \& B. Kolarevic, Eds.) (Vol. 1). New York, NY: Routledge.
Kronenburg, R. (2015). Building Dynamics: Exploring Architecture of Change. (B. Kolarevic \& V. Parlac, Eds.) (Vol. 1). New York, NY: Routledge.

Indrawati, S. M., \& Steer, A. (2005). Tsunami Evaluation Coalition Other Evaluations and Reviews - Country Case Studies (p. i). Jakarta, Indonesia: nternational Donor Community, BAPPENAS, Retrieved from http://www.tsunamievaluation.org/Other+Evaluations+and+Reviews/Country+Case+ Studies.htm

Insurance Council of New Zealand. (2016, May 6). Rebuild Statistics ICNZ. Retrieved 26 June 2016, from http://www.icnz.org.nz/natural-disaster/canterbury/rebuildstatistics/

Suaris, W., \& Khan, M. S. (1995). Residential Construction Failures Caused by Hurricane Andrew. Journal of Performance of Constructed Facilities, 9(1), 24.

Tibbits, S. (2015). Building Dynamics: Exploring Architecture of Change. (B. Kolarevic \& V. Parlac, Eds.) (Vol. 1). New York, NY: Routledge.

Van De Lindt, J. W., Graettinger, A., Gupta, R., Skaggs, T., Pryor, S., \& Fridley, K. J. (2007). Performance of Wood-Frame Structures during Hurricane Katrina. Journal of Performance of Constructed Facilities, 21(2), 108-116. 